\title{
Effects of cyanocobalamin and its combination with morphine on neuropathic rats and the relationship between these effects and thrombospondin-4 expression
}

\author{
Neslihan Düzenli ${ }^{1}$, Sibel Ülker $^{1}$, Gülgün ŞengülI $^{2}$, Buse Kayhan $^{3}$, and Aytül Önal ${ }^{1}$ \\ 'Department of Medical Pharmacology, Faculty of Medicine, Ege University, Bornova, Izmir, Turkey \\ ${ }^{2}$ Department of Anatomy, Faculty of Medicine, Ege University, Bornova, Izmir, Turkey \\ ${ }^{3}$ Department of Neuroscience, Faculty of Health Sciences, Ege University, Bornova, Izmir, Turkey
}

Received September 16, 2021

Revised December 4, 2021

Accepted December 4, 2021

Handling Editor: Sang Hun Kim

\author{
Correspondence \\ Neslihan Düzenli \\ Department of Medical Pharmacology, \\ Faculty of Medicine, Izmir Democracy \\ University, Mehmet Ali Akman, 13. Sk. \\ No:2, 35290 Konak/Izmir, Turkey \\ Tel: +90-232-260-1001 (630) \\ Fax: +90-232-260-1004 \\ E-mail: neslihan.duzenli@idu.edu.tr
}

Neslihan Düzenli's current affiliation Department of Medical Pharmacology, Faculty of Medicine, Izmir Democracy University, Konak 35290, Izmir, Turkey
Background: Thrombospondin-4 (TSP4) upregulates in the spinal cord following peripheral nerve injury and contributes to the development of neuropathic pain (NP). We investigated the effects of cyanocobalamin alone or in combination with morphine on pain and the relationship between these effects and spinal TSP4 expression in neuropathic rats.

Methods: NP was induced by chronic constriction injury (CCl) of the sciatic nerve. Cyanocobalamin ( 5 and $10 \mathrm{mg} / \mathrm{kg} /$ day) was administered 15 days before $\mathrm{CCl}$ and then for 4 and 14 postoperative days. Morphine $(2.5$ and $5 \mathrm{mg} / \mathrm{kg} /$ day $)$ was administered only post-CCl. Combination treatment included cyanocobalamin and morphine, 10 and $5 \mathrm{mg} / \mathrm{kg} /$ day, respectively. All drugs were administered intraperitoneally. Nociceptive thresholds were detected by esthesiometer, analgesia meter, and plantar test, and TSP4 expression was assessed by western blotting and fluorescence immunohistochemistry.

Results: $\mathrm{CCl}$ decreased nociceptive thresholds in all tests and induced TSP4 expression on the 4th postoperative day. The decrease in nociceptive thresholds persisted except for the plantar test, and the increased TSP4 expression reversed on the 14th postoperative day. Cyanocobalamin and low-dose morphine alone did not produce any antinociceptive effects. High-dose morphine improved the decreased nociceptive thresholds in the esthesiometer when administered alone but combined with cyanocobalamin in all tests. Cyanocobalamin and morphine significantly induced TSP4 expression when administered alone in both doses for 4 or 14 days. However, this increase was less when the two drugs are combined.

Conclusions: The combination of cyanocobalamin and morphine is more effective in antinociception and partially decreased the induced TSP4 expression compared to the use of either drug alone.

Key Words: Analgesics, Opioid; Cobamides; Morphine; Neuralgia; Pain; Peripheral Nerve Injuries; Rats; Sciatic Nerve; Spinal Cord; Thrombospondins; Vitamin B 12. (c) This is an open-access article distributed under the terms of the Creative Commons Attribution Non-Commercial License (http://creativecommons.org/licenses/by-nc/4.0/), which permits unrestricted non-commercial use, distribution, and reproduction in any medium, provided the original work is properly cited.

(C) The Korean Pain Society, 2022
Author contributions: Neslihan Düzenli: Study conception, Data acquisition, Data analysis, Writing/manuscript preparation; Sibel Ülker: Writing/ manuscript preparation; Gülgün Şengül: Investigation, Data acquisition; Buse Kayhan: Investigation, Data acquisition; Aytül Önal: Data analysis, Supervision. 


\section{INTRODUCTION}

Neuropathic pain (NP) is pain caused by a lesion or disease affecting the somatosensory nervous system [1]. NP may be caused by diverse conditions such as diabetic neuropathy and spinal cord injury [2]. Currently, antidepressants, anticonvulsants, topical and narcotic analgesics are being used to treat NP [3]. However, none of these drugs are spesific for NP treatment, and their effectiveness is questionable. Moreover, many of them have a short duration of effectiveness, limited safety, and many adverse effects [4]. For such reasons, the treatment of NP is complicated and often inadequate.

Thrombospondins (TSPs) are oligomeric, multidomain, calcium-binding extracellular glycoproteins [5]. TSPs are expressed primarily during development and growth, and in response to injury [6], with critical roles in abnormal synaptogenesis, leading to spinal sensitization and pain [7]. Peripheral nerve injury induces TSP4 upregulation in the dorsal spinal cord that correlates with behavioral hypersensitivity initiation and maintenance. In rats, blockage of injury-induced upregulation of TSP4 expression prevented and reversed behavioral hypersensitivity development $[7,8]$. Therefore, it is accepted that blocking TSP4 expression has therapeutic potential in the targeted therapy of NP.

Opioid analgesics are frequently preferred to treat acute and chronic pain states, including, especially, cancer and NP. Although they are highly effective for treating pain, their intensely addictive effects and the development of tolerance to their antinociceptive effects during long-term treatment [9] considerably restrict the utilization of opiates. Vitamin $B_{12}$ is involved in the metabolic functions of every body cell, i.e., deoxyribonucleic acid synthesis and regulation, fatty acid metabolism, and energy production [10]. It is a neurotrophic agent with a particular affinity to neural tissues [11]. There is much evidence that vitamin $B_{12}$ supports axonal cell myelinization and peripheral nerve regeneration by various mechanisms during the injury or inflammation of peripheral nerves [12]. It has been suggested that vitamin $B_{12}$ exerts its antiallodynic effect by inhibiting peripheral pain signals; however, the mechanisms underlying its analgesic effect are still not fully elucidated [13]. Combining two or more drugs in patients with NP who cease treatment due to adverse effects or other reasons may allow lower doses [14], improve analgesic efficacy, and reduce adverse effects [15]. Animal studies suggest that vitamin $B_{12}$ may provide an opioid-sparing effect when combined with opiates, allowing the reduction of the opioid dose [16]. However, there is no consensus on this issue or events mediating this effect. TSP4 may be a potential candidate composing one of the fundamental mechanisms in NP initiation and maintenance. There is very little information about the participation of TSP4 in the mechanisms of action of drugs or drug combinations in NP treatment.

Therefore, we aimed to investigate whether the administration of cyanocobalamin (a vitamin $\mathrm{B}_{12}$ analogue) alone or in combination with morphine (an opiate analgesic) induces an antinociceptive effect in neuropathic rats, and reveal the relationship between lumbar spinal cord TSP4 level and this potential antinociceptive effect.

\section{MATERIALS AND METHODS}

\section{Animals}

Adult Wistar rats of either sex $(\mathrm{n}=132,200-300 \mathrm{~g})$ were obtained from Ege University Center for Research on Laboratory Animals. The experimental protocol was approved by the Ege University Animal Experiments Local Ethics Committee (approval No. 2017-092) and performed according to the Guide for the Care and Use of Laboratory Animals approved by the National Institutes of Health of the United States. The animals were housed in each plastic cage covered with sawdust under a standard 12 hours light/12 hours dark cycle in a room maintained at $24^{\circ} \mathrm{C} \pm 1^{\circ} \mathrm{C}$ and were given food and water ad libitum.

\section{Induction of NP}

NP was induced in rats by a slight modification of the chronic constriction injury (CCI) model of Bennett and Xie [17]. Silk suture was used instead of catgut as the binding material. Briefly, the right sciatic nerves of rats were exposed under ketamine/xylazine (40:20 mg/kg, intraperitoneal [i.p.]) anesthesia and loosely ligated three times with a 4.0 silk suture leaving approximately $1 \mathrm{~mm}$ spaces in between. The muscle, fascia, and skin were sutured with 3.0 silk. Identical surgery was also performed on sham animals without ligating the sciatic nerve. After surgery, the rats were returned to their cages. No surgical procedure was administered to the control animals.

\section{Experimental groups and administration of drugs}

The rats were divided into six experimental groups. Following administration of cyanocobalamin or saline as a pre-emptive treatment for 15 days, saline, cyanocobalamin (Deva Holding A.S., Kocaeli, Turkey), and/or morphine (Galen Ltd., Istanbul, Turkey) were administered for 4 and 14 postoperative days. All drugs were administered by intraperitoneal injection. Fig. 1 shows the flowchart of 

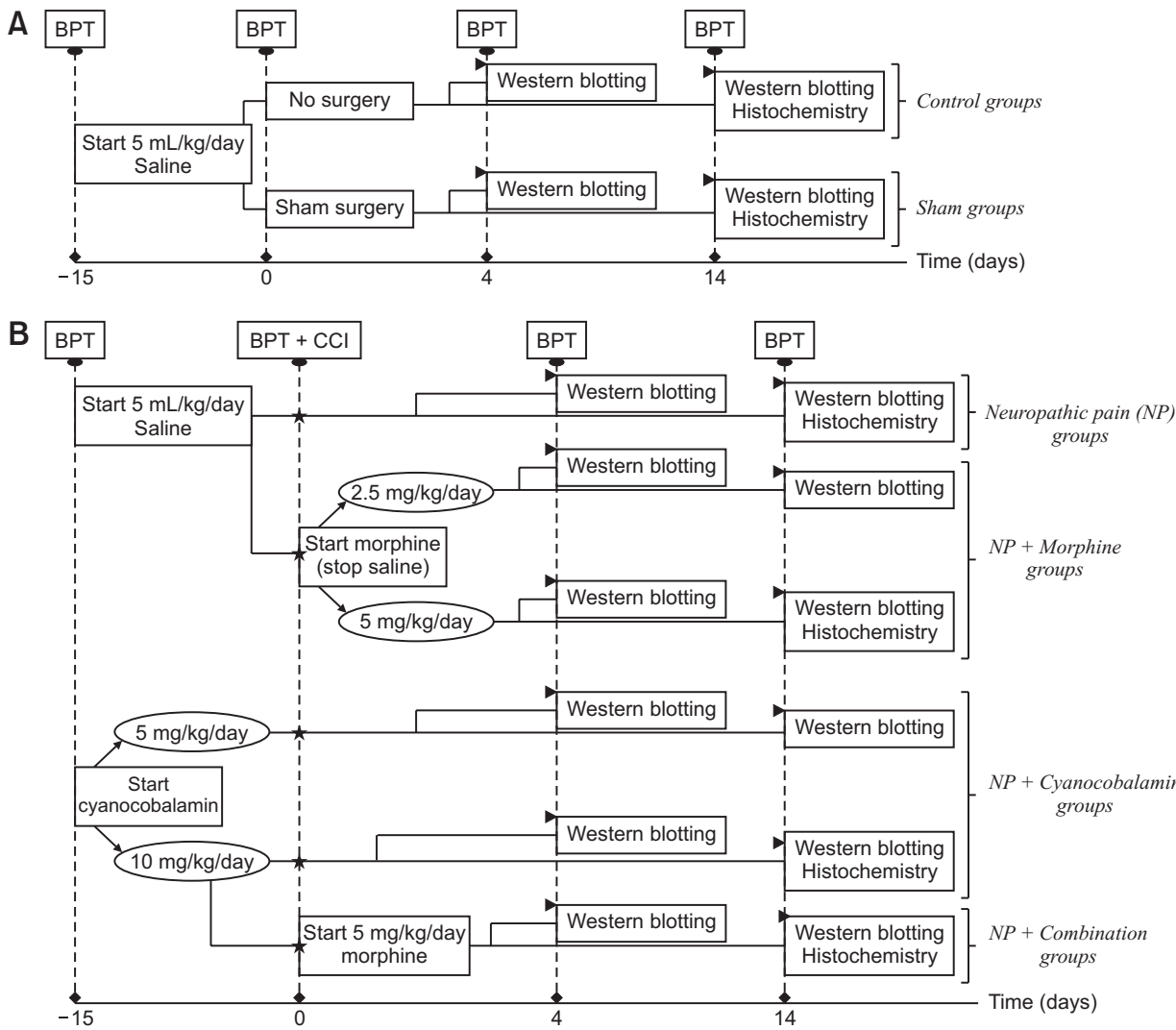

Fig. 1. Flowchart showing the experimental design of the present study. (A) Experimental groups without $\mathrm{CCl}$ and (B) experimental groups with $\mathrm{CCl} /$ neuropathic pain. Asterisk $(\star)$ represent CCl. Arrowhead $(\downarrow)$ indicates the sacrification and the subsequent process. Notes: 1) The pain thresholds of all animals, including rat groups sacrificed on the 14th postoperative day were measured on the 4th postoperative day and these data were also used to assess pain threshold change on the 4th postoperative day. 2) Spinal cord tissues used in Western Blotting and Fluorescence Immunohistochemistry studies were obtained from separate animals. CCl: chronic construction injury of sciatic nerve, BPT: behavioral pain tests. the experimental design.

\section{Behavioral pain tests}

Behavioral pain tests were carried out before starting injection (basal paw nociceptive threshold) and before surgery (preoperative paw nociceptive threshold) on the 4 th and 14 th postoperative days. All rats were habituated to the test environment for approximately 30 minutes before the experiments; meanwhile, food and water were withdrawn. A dynamic plantar esthesiometer (Ugo Basile, Gemonio, Italy), plantar test (Ugo Basile), and analgesia meter (Ugo Basile) were used to measure mechanical allodynia, thermal hyperalgesia, and mechanical hyperalgesia, respectively. Nociceptive thresholds in the analgesia meter were expressed in grams as the force required to elicit a withdrawal response. In the dynamic plantar esthesiometer and plantar tests, the paw withdrawal latencies were expressed in seconds as the endurance duration of the rat to mechanical and thermal stimuli. Cut-off values were accepted as $50 \mathrm{~g}$ force and 20 seconds to prevent skin sensitization and thermal injury, respectively. Stimuli were administered a minimum of 3 and maximum of 5 times to each paw with 5 -min intervals in between. The data were averaged and expressed as the percentage change in the threshold, which was calculated using the following formula:
$\%$ change in nociceptive threshold $=$

(pre- or post-surgery paw withdrawal latency/

basal paw withdrawal latency) $\times 100$

\section{Western blot analysis in the lumbar spinal cord}

Spinal cord segments from L4-6 were removed from rats under anesthesia (ketamine/xylazine; $40 / 20 \mathrm{mg} / \mathrm{kg}$, i.p.) on the 4 th and 14th postoperative days. The samples (100 $\mathrm{mg} / \mathrm{mL}$ ) were homogenised in lysis buffer (20 mM Trishydrochloric acid [HCL], $3 \mathrm{mM}$ ethylene glycol tetraacetic acid [EGTA], $5 \mathrm{mM}$ ethylene diamine tetraacetic acid [EDTA], $10 \mathrm{mM}$ dithiothreitol [DTT], $0.5 \mathrm{mg} / \mathrm{mL}$ aprotinin, $0.001 \mathrm{mg} / \mathrm{mL}$ pepstatin, $0.001 \mathrm{mg} / \mathrm{mL}$ leupeptin, and 0.5 $\mathrm{mM}$ phenylmethylsulfonyl fluoride [PMSF] in $\mathrm{pH}$ : 7.5) on ice, then centrifuged at $1,400 \mathrm{rpm}$ at $4^{\circ} \mathrm{C}$ for 30 minutes to remove insoluble pellets. The supernatants were removed, and protein concentration was measured at $750 \mathrm{~nm}$ wavelength in the spectrophotometer by the Lowry method [18]. One hundred $\mu$ g of protein were mixed with $4 \times$ Laemmeli buffer (pH: 8) and kept at $100^{\circ} \mathrm{C}$ for 2 minutes, and loaded onto $4 \%$ stacking and $10 \%$ separating sodium dodecyl sulphate-polyacrylamide gel electrophoresis (SDS-PAGE) gel. The resolved proteins were transferred onto the nitrocellulose membrane. Blots were blocked for an hour at room temperature with $1 \%$ bovine serum albumin and then incubated overnight at $4^{\circ} \mathrm{C}$ with antibodies against TSP 4 
(1:250, Santa Cruz Biotechnology, Dallas, TX) and $\beta$-actin (1:20,000, Sigma-Aldrich Corp., St. Louis, MO). After extensive washing, the membranes were incubated with a secondary antibody (1:500 for TSP4 and 1:10,000 for $\beta$-actin, GE Healthcare UK Limited, Buckinghamshire, UK) in blocking buffer for 1 hour at $37^{\circ} \mathrm{C}$. Finally, the membranes were incubated with freshly prepared electrochemiluminescence solution (GE Healthcare UK Limited, Amersham, UK) for 5 minutes in the darkroom. Band images were obtained by scanning with a chemiluminescence device (LI-COR C-DIGIT Blot Scanner, Model 3600; LI-COR, Inc., Lincoln, NE). The band densities were quantified using the Image Studio Digits v4.0 program (LI-COR, Inc.). The data were presented as the ratio of TSP4 band density over $\beta$-actin band density.

\section{Fluorescence immunohistochemistry}

On the 14th postoperative day, intracardiac perfusion with phosphate buffered saline (PBS, $0.01 \mathrm{M}$ ) followed by a $4 \%$ cold paraformaldehyde (PFA) solution was carried out in rats under anesthesia. The lumbar segments of the spinal cord (L4-L6) were removed by laminectomy and post-fixated overnight in 4\% PFA solution and then transferred into $30 \%$ sucrose until sectioning. Thirty $\mu \mathrm{m}$ thick transverse spinal sections were obtained using the LEICA CM1950 cryostat device (Leica Microsystems Inc., Wetzlar, Germany), and sections were placed on polylysine-coated slides.

Sections were blocked with PBS containing $1 \%$ bovine serum albumin and Triton $\mathrm{X}$ for an hour at room temperature and incubated with primary antibodies TSP4 (mouse monoclonal, 1:100, Santa Cruz Biotechnology) and glial fibrillary acidic protein (GFAP; rabbit polyclonal, 1:100, Abcam, Cambridge, UK) overnight at $4^{\circ} \mathrm{C}$ and then with secondary antibodies (goat anti-mouse polyclonal immunoglobulin $\mathrm{G}[\mathrm{IgG}]$, fluorescein conjugate, 1:100, EMD Millipore Corporation, Temecula, CA, and goat anti-rabbit IgG $[\mathrm{H}+\mathrm{L}]$, rhodamine conjugate, 1:100, EMD Millipore Corporation) for 2 hours at room temperature in the dark. The sections were coverslipped using $90 \%$ glycerol. The stained sections were examined under a fluorescence microscope (Olympus BX51; Olympus, Tokyo, Japan) and images were recorded and analyzed using a computerized image-analyzer program, Image J (National Institutes of Health, Bethesda, MD). The immunoreactivity of TSP4 protein in the dorsal horn of the lumbar spinal cord was defined as \% surface area, and astrocyte cells were quantified as the GFAP (+) cell count.

\section{Statistical analysis}

Basal and preoperative nociceptive threshold values were compared with the independent-samples $t$-test, and \% changes in nociceptive thresholds values on the 4 th and 14th postoperative days were evaluated using one-way analysis of variance (ANOVA) followed by a posthoc Bonferroni test. Data obtained from densitometric analysis of protein bands were evaluated using the Kruskal-Wallis test followed by the Mann-Whitney $U$-test. Data obtained from the immunostained sections on the 14th postoperative day were analyzed with one-way ANOVA followed by a posthoc Least Significant Difference (LSD) test. The Pearson correlation coefficient was performed to determine the relationship between the GFAP (+) cell number and the percentage of surface area of TSP4 protein in the dorsal horn of the lumbar spinal cord on the 14th postoperative day. IBM SPSS Statistics 25.0 (IBM Co., Armonk, NY) was used to perform statistical analyzes. Data were presented as mean \pm standard error of mean and the significance level was accepted as $P<0.05$.

\section{RESULTS}

\section{Assessment of behavioral pain tests}

Nociceptive thresholds of both paws were measured before starting the injection (the basal paw nociceptive threshold) and just before surgery (the preoperative paw nociceptive threshold) to determine whether nociceptive thresholds were affected by pre-emptive cyanocobalamin or saline administration for 15 consecutive days. There were no significant differences between basal and preoperative nociceptive threshold values in all three tests. Therefore, the data obtained from behavioral pain tests were presented as the percentage of postoperative nociceptive threshold values over the basal values.

Only the data obtained from the lesioned (right) paws were presented here, since each experimental group's non-lesioned (left) paw nociceptive threshold remained unchanged compared to its basal value throughout the experiment, and there were no differences between the groups in all three tests. There were no differences between the nociceptive thresholds of the control and sham groups in any tests (Fig. 2, 3); therefore, the postoperative nociceptive threshold of each NP group was compared with that of the sham group. Also, postoperative nociceptive thresholds of NP groups were compared among themselves.

Ligation of the sciatic nerve decreased the nociceptive thresholds remarkably on the 4th postoperative day in all 

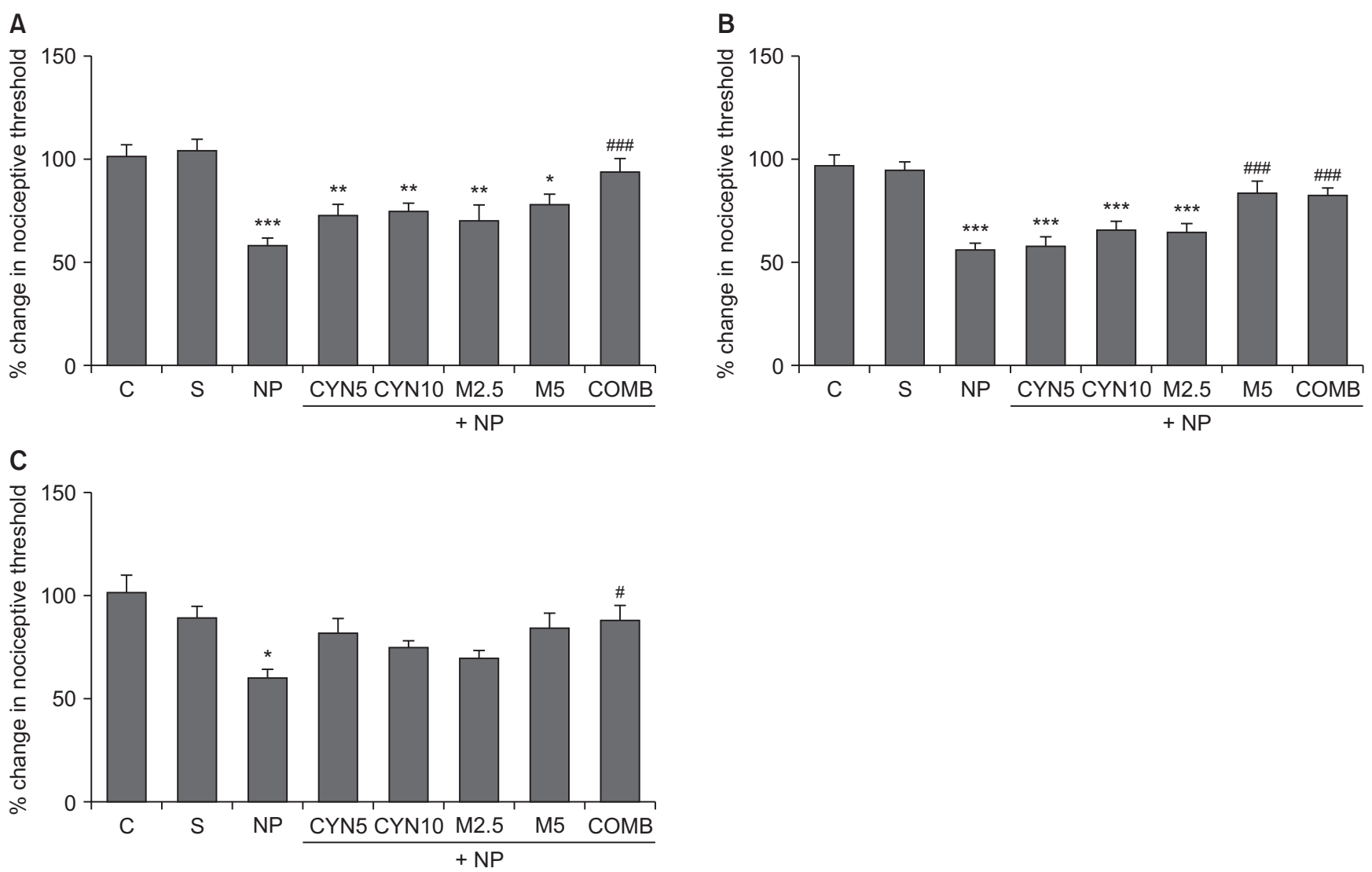

Fig. 2. Nociceptive thresholds of lesioned paws were expressed on the 4th postoperative day as the percentage change in the threshold, calculated using the following formula: \% change in nociceptive threshold $=($ Postoperative paw withdrawal latency/Basal paw withdrawal latency $) \times 100$. Mechanical hyperalgesia, tactile allodynia, and thermal hyperalgesia are shown by using (A) analgesia meter, (B) dynamic plantar esthesiometer, and (C) plantar test. Statistical analyzes of data obtained from behavioral pain tests were performed using one-way analysis of variance followed by posthoc Bonferonni test $(\mathrm{n}=11-20)$. Data are presented as mean \pm standard error of mean. (A) Analgesia meter. $* * * P<0.001, * * P=0.008, * * P=0.006, * * P=0.003, * P=$ 0.018; NP, CYN5, CYN10, M2.5, and M5, respectively, vs. S group. ${ }^{\# \#} P<0.001$, COMB vs. NP group. (B) Dynamic plantar esthesiometer. $* * * P<0.001$, $* * * P<0.001, * * * P<0.001, * * * P<0.001$; NP, CYN5, CYN10, and M2.5, respectively, vs. S group. ${ }^{\# \#} P<0.001, \# \# P<0.001$; M5 and COMB, respectively, vs. NP group. (C) Plantar test. ${ }^{*} P=0.016$, NP vs. S group. ${ }^{\#} P=0.030$, COMB vs. NP group. C: control, S: Sham, NP: saline-injected neuropathic pain, CYN5 and CYN10: NP + 5 and $10 \mathrm{mg} / \mathrm{kg} /$ day cyanocobalamin, respectively, M2.5 and M5: NP + 2.5 and $5 \mathrm{mg} / \mathrm{kg} /$ day morphine, respectively, COMB: $\mathrm{NP}+10 \mathrm{mg} / \mathrm{kg} /$ day cyanocobalamin $+5 \mathrm{mg} / \mathrm{kg} /$ day morphine.

tests $(P<0.001$, Fig. 2A, B; $P=0.016$, Fig. 2C; saline-injected NP group vs. $S$ group). Similarly, there was still a reduction in mechanical nociceptive thresholds on the 14th postoperative day $(P<0.001$, Fig. 3A, B; saline-injected NP group vs. $S$ group); however, there were no significant differences between the thermal nociceptive threshold of all groups (Fig. 3C).

In comparison to the saline-injected NP group, cyanocobalamin at both doses and low dose morphine $(2.5 \mathrm{mg} /$ $\mathrm{kg} /$ day) failed to improve reduced nociceptive thresholds on the 4th (Fig. 2) and 14th (Fig. 3A, B) postoperative days when they were administered alone. However, high dose morphine $(5 \mathrm{mg} / \mathrm{kg} /$ day $)$ alone noticeably reversed the reduced mechanical nociceptive thresholds measured by the dynamic plantar esthesiometer, but not the analgesia meter (Fig. 2A, 3A, M5 group vs. saline-injected NP group, $)$, on the 4 th $(P<0.001$, Fig. $2 B$, M5 group vs. saline- injected NP group) and 14th ( $P=0.014$, Fig. 3B, M5 group vs. saline-injected NP group) postoperative days. The combination of cyanocobalamin and morphine (10 and $5 \mathrm{mg} / \mathrm{kg} /$ day, respectively) exhibited remarkable antihyperalgesic and antiallodynic effects by increasing the decreased nociceptive thresholds in all tests on the 4 th postoperative day when compared to the saline-injected NP group ( $P<0.001$, Fig. 2A, B; $P=0.030$, Fig. 2C). Also, its antihyperalgesic and antiallodynic effects (measured by the analgesia meter and esthesiometer, respectively) continued on the 14th postoperative day $(P=0.002$, Fig. 3A; $P=$ 0.006, Fig. 3B, COMB group vs. saline-injected NP group).

\section{Assessment of TSP4 protein expression in the lumbar spinal cord}

There were no differences in TSP4 expression between 

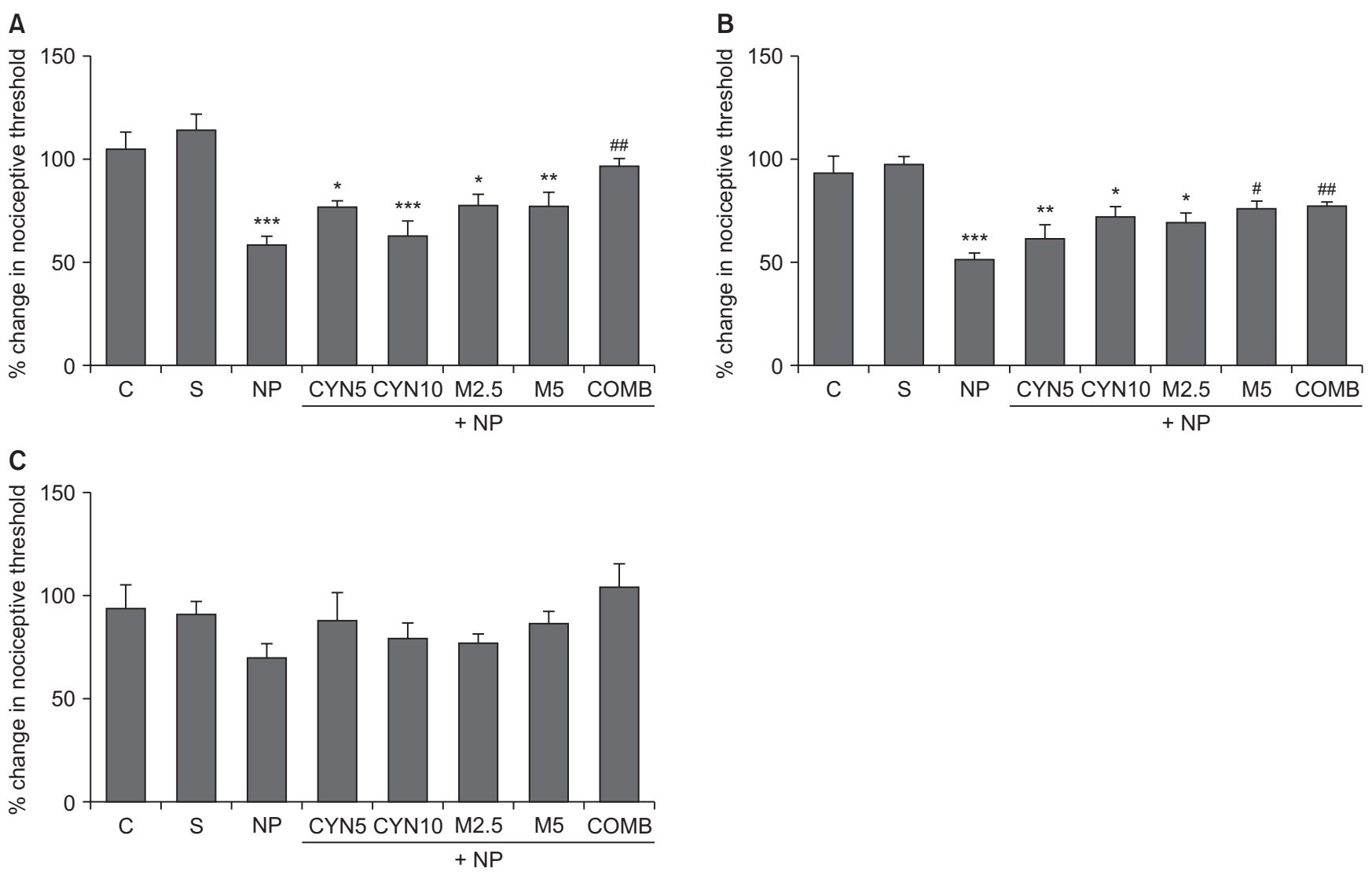

Fig. 3. Nociceptive thresholds of lesioned paws were expressed on the 14th postoperative day as the percentage change in the threshold, calculated using the following formula: \% change in nociceptive threshold $=($ Postoperative paw withdrawal latency/Basal paw withdrawal latency $) \times 100$. Mechanical hyperalgesia, tactile allodynia, and thermal hyperalgesia are shown by using (A) analgesia meter, (B) dynamic plantar esthesiometer, and (C) plantar test. Statistical analyzes of data obtained from behavioral pain tests were performed using one-way analysis of variance followed by posthoc Bonferonni test $(\mathrm{n}=7$-14). Data are presented as mean \pm standard error of mean. (A) Analgesia meter. $* * * P<0.001, * P=0.021, * * * P<0.001, * P=0.015, * * P=$ 0.005 ; NP, CYN5, CYN10, M2.5, and M5, respectively, vs. S group. ${ }^{\# \#} P=0.002$, COMB vs. NP group. (B) Dynamic plantar esthesiometer. $* * * P<0.001$, ${ }^{*} P=0.001,{ }^{*} P=0.019,{ }^{*} P=0.016$; NP, CYN5, CYN10, and M2.5, respectively, vs. S group. ${ }^{\#} P=0.014,{ }^{\# \#} P=0.006$; M5 and COMB, respectively, vs. NP group. C: control, S: Sham, NP: saline-injected neuropathic pain, CYN5 and CYN10: NP + 5 and $10 \mathrm{mg} / \mathrm{kg} /$ day cyanocobalamin, respectively, M2.5 and M5: NP + 2.5 and $5 \mathrm{mg} / \mathrm{kg} /$ day morphine, respectively, COMB: NP + $10 \mathrm{mg} / \mathrm{kg} /$ day cyanocobalamin $+5 \mathrm{mg} / \mathrm{kg} /$ day morphine.

control and sham groups; therefore, spinal cord TSP4 expressions in all NP groups were compared initially with the sham group and then with each other on the 4th and 14th postoperative days. Our results showed that spinal cord TSP4 expressions of all NP groups increased on the 4th postoperative day compared to the sham group $(P=$ 0.004, saline-injected NP group; $P=0.009$, CYN5 group; $P$ $=0.019$, CYN10 group; $P=0.006$, M2.5 group; $P=0.019$, M5 group; and $P=0.002$, COMB group, respectively, Fig. 4A). However, there were no significant differences among all NP groups on the 4th postoperative day (Fig. 4A).

While reviewing the results of the 14th postoperative day, it was detected that the TSP4 expression of the salineinjected NP group was statistically not different from the sham group (Fig. 4B). However, in all drug administered NP groups, TSP4 expression significantly increased compared to the sham group $(P=0.007$, CYN5 and CYN10 groups; $P=0.031$, M2.5 group; $P=0.007$, M5 group; and $P$
$=0.039, \mathrm{COMB}$ group, respectively, Fig. 4B). This increase was quite remarkable in the NP rats treated with both doses of cyanocobalamin alone compared to the salineinjected NP group ( $P=0.015$, CYN5 group; and $P=0.031$, CYN10 group, respectively, Fig. 4B).

\section{Assessment of TSP4 and GFAP immunoreactivities in the lumbar spinal cord}

Representative images of immunostainings of TSP4 and GFAP in the lumbar spinal cord sections on the 14th postoperative day are presented in Fig. 5A and 5B, respectively. There were no statistically significant differences between TSP4 and GFAP immunoreactivities of control, sham, and saline-injected NP groups in the ipsilateral (injury side) and contralateral (non-injury side) dorsal horn (Fig. 6). Therefore, the data of drug-administered NP groups were compared to that of the saline-injected NP group. Our re- 

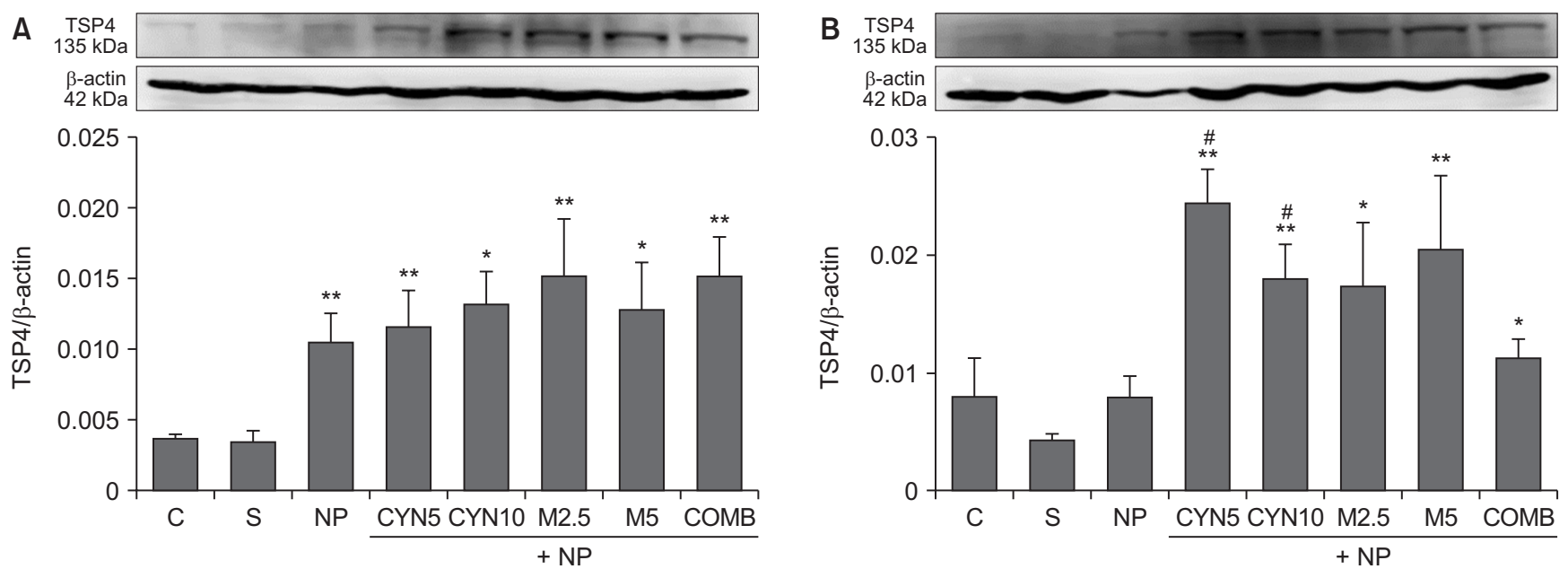

Fig. 4. Thrombospondin-4 (TSP4) protein expressions in L4-L6 spinal cord segments taken from rats on the (A) 4th and (B) 14th postoperative days. Bar graphs indicate results of densitometric analysis of the TSP4 protein (135 kDa) bands as normalized to the quantity of $\beta$-actin (42 kDa) protein. Statistical analyses of densitometric data of protein bands obtained from Western blotting were performed using the Kruskal-Wallis test followed by the MannWhitney $U$-test $(n=4-6)$. Data are presented as mean \pm standard error of mean. $(A) * * P=0.004, * * P=0.009, * P=0.019, * * P=0.006, * P=0.019$, $\star * P=0.002$; NP, CYN5, CYN10, M2.5, M5, and COMB, respectively, vs. S group. (B) $* * P=0.007, * * P=0.007, * P=0.031, * * P=0.007, * P=0.039$; CYN5, CYN10, M2.5, M5, and COMB, respectively, vs. S group. ${ }^{\#} P=0.015,{ }^{\#} P=0.031$; CYN5 and CYN10, respectively, vs. NP group. C: control, S: Sham, NP: saline-injected neuropathic pain, CYN5 and CYN10: NP + 5 and $10 \mathrm{mg} / \mathrm{kg} /$ day cyanocobalamin, respectively, M2.5 and M5: NP + 2.5 and 5 mg/kg/ day morphine, respectively, COMB: NP $+10 \mathrm{mg} / \mathrm{kg} /$ day cyanocobalamin $+5 \mathrm{mg} / \mathrm{kg} /$ day morphine.

A

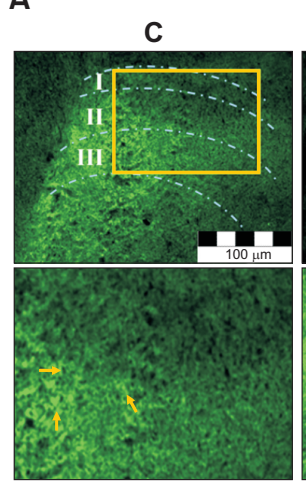

B

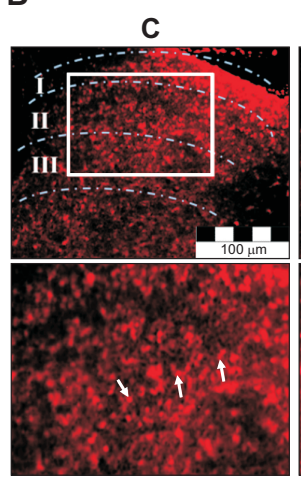

s

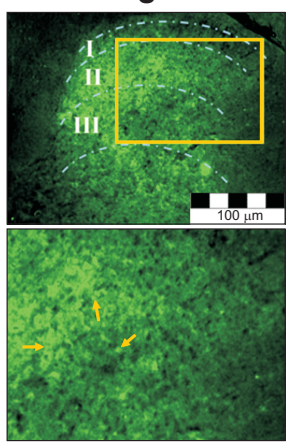

S

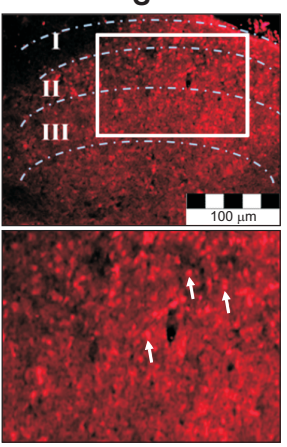

NP

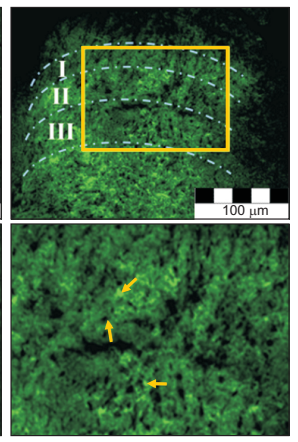

NP

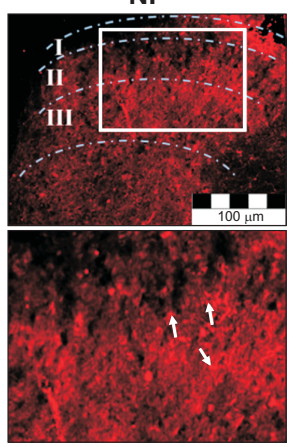

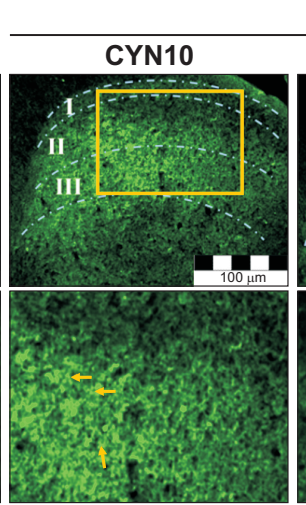
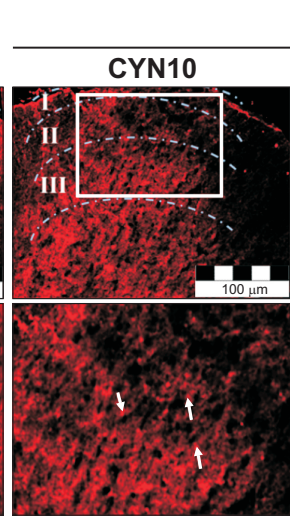

+ NP

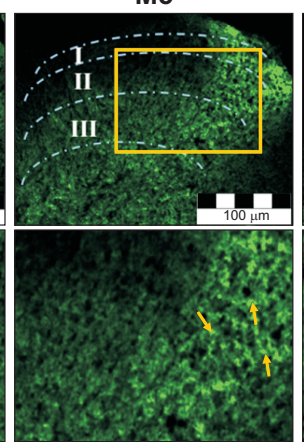

+ NP

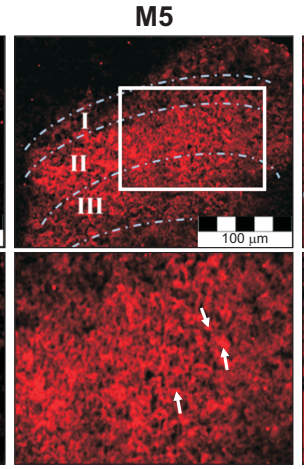

\section{COMB}

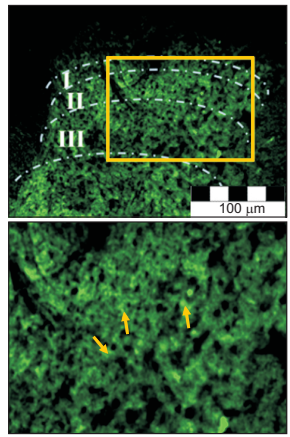

COMB

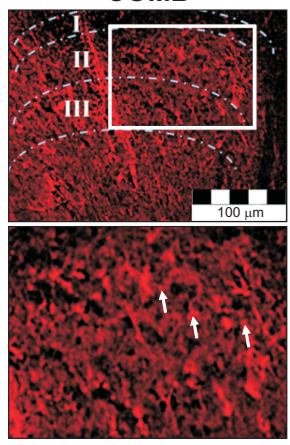

Fig. 5. Representative images of (A) thrombospondin-4 (TSP4) and (B) glial fibrillary acidic protein (GFAP) immunoreactivities in the ipsilateral dorsal horns of L4-L6 spinal cord transverse sections of experimental groups (Scale bar: $100 \mu \mathrm{m}$ ). The analyzed cross-sectional areas contain lamina I-II-III in the dorsal horn and have been marked over the images. The areas marked in the above images are magnified just below, and TSP4 proteins are indicated by yellow arrows and GFAP immunoreactive cells by white arrows. C: control, S: Sham, NP: saline-injected neuropathic pain, CYN10: NP + 10 mg/ $\mathrm{kg} /$ day cyanocobalamin, M5: NP + $5 \mathrm{mg} / \mathrm{kg} /$ day morphine, COMB: $\mathrm{NP}+10 \mathrm{mg} / \mathrm{kg} /$ day cyanocobalamin $+5 \mathrm{mg} / \mathrm{kg} /$ day morphine. 

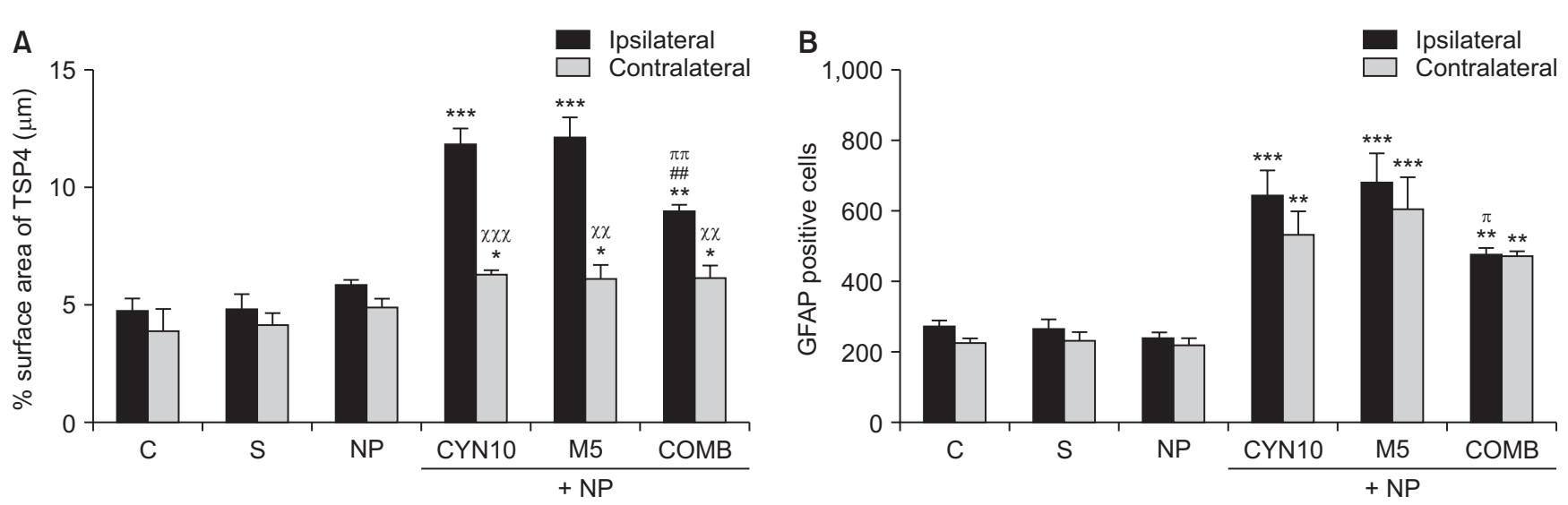

Fig. 6. (A) Percentage of thrombospondin-4 (TSP4) surface area $(\mu \mathrm{m})$ and (B) glial fibrillary acidic protein (GFAP) immunoreactive cells (number) in the lumbar dorsal horn on the 14th postoperative day. The analyzed cross-sectional areas contain lamina I-II-III in the dorsal horn. Statistical analyzes of data obtained from immunostained sections were performed using one-way analysis of variance followed by a posthoc LSD test $(n=3-4)$. Each bar represents the mean \pm standart error of the mean. (A) $* * * P<0.001, * * * P<0.001, * * P=0.001$; ipsilateral sides of CYN10, M5, and COMB, respectively, vs. ipsilateral side of NP group. ${ }^{\# \#} P=0.001$, ipsilateral side of COMB vs. ipsilateral side of CYN10 group. ${ }^{\mathrm{m}} P=0.002$, ipsilateral side of COMB vs. ipsilateral side of M5 group. ${ }^{*} P=0.017,{ }^{*} P=0.021,{ }^{*} P=0.022$; contralateral sides of CYN10, M5, and COMB, respectively, vs. contralateral side of NP group. ${ }^{x \times x} P$ $<0.001,{ }^{x x} P=0.006,{ }^{x x} P=0.004$; contralateral sides of CYN10, M5, and COMB, respectively, vs. own ipsilateral sides. (B) $* * * P<0.001$, $* * * P<0.001$, $* * P=0.005$; ipsilateral sides of CYN10, M5, and COMB, respectively, vs. ipsilateral side of NP group. $\Pi P=0.029$, ipsilateral side of COMB vs. ipsilateral side of M5 group. $* * P=0.001, * * * P<0.001, * * P=0.005$; contralateral sides of CYN10, M5, and COMB, respectively, vs. contralateral side of NP group. C: control, S: Sham, NP: Saline-injected neuropathic pain, CYN10: NP + 10 mg/kg/day cyanocobalamin, M5: NP + 5 mg/kg/day morphine, COMB: $\mathrm{NP}+10 \mathrm{mg} / \mathrm{kg} /$ day cyanocobalamin $+5 \mathrm{mg} / \mathrm{kg} /$ day morphine.
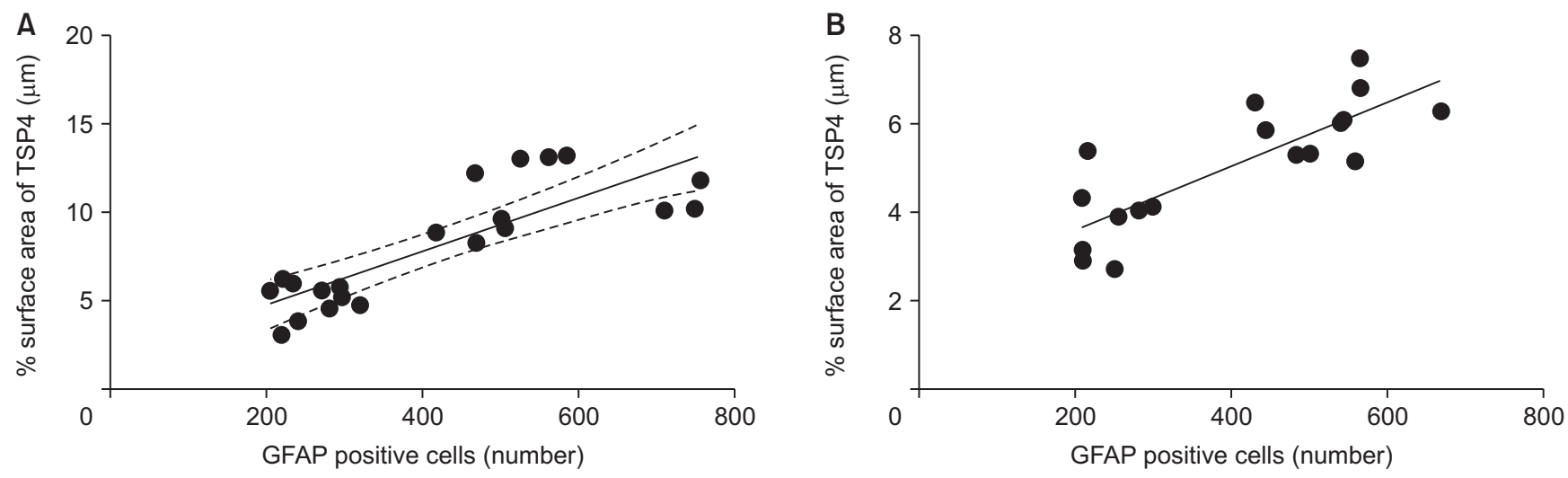

Fig. 7. Pearson's correlation shows a linear relationship between glial fibrillary acidic protein (GFAP) immunoreactive cells and thrombospondin-4 (TSP4) protein surface area in both $(A)$ ipsilateral $(P<0.001, r=0.826, n=21)$ and $(B)$ contralateral $(P<0.001, r=0.811, n=18)$ spinal cord sections. The data of each group show a normal distribution pattern. GFAP positive cell number is accepted as the dependent variable (X-axis), and the percentage of TSP4 surface area is accepted as the independent variable (Y-axis).

sults showed that TSP4 and GFAP immunoreactivities of drug-administered groups increased remarkably at both ipsilateral (for TSP4, $P<0.001, P<0.001, P=0.001$, Fig. 6A; for GFAP, $P<0.001, P<0.001, P=0.005$, Fig. 6B; CYN10, M5, and COMB groups vs. saline-injected NP group, respectively) and contralateral sides (for TSP4, $P=0.017, P=0.021$, $P=0.022$, Fig. 6A; for GFAP, $P=0.001, P<0.001, P=0.005$, Fig. 6B, CYN10, M5, and COMB groups vs. saline-injected NP group, respectively).

However, the increase in ipsilateral TSP4 immunoreactivity in the combination group was significantly less than those in the cyanocobalamin and morphine alone groups
$(P=0.001$ and $P=0.002$, COMB group vs. CYN10 and M5 groups, respectively, Fig. 6A). In comparison, the increase in ipsilateral GFAP immunoreactivity in the combination group was less than only that of the morphine-injected NP group ( $P=0.029$, COMB group vs. M5 group, Fig. $6 B$ ).

Additionally, it was noticed that in all drug-administered NP groups, increase in TSP4 immunoreactivity in the ipsilateral sides was significantly higher than their contralateral sides $(P<0.001, P=0.006, P=0.004$; contralateral side of CYN10, M5, and COMB groups vs. their ipsilateral side, respectively, Fig. 6A); however, this was not the case for ipsilateral and contralateral GFAP immunoreactivities 
in these groups (Fig. 6B).

Pearson correlation showed a very strong linear relationship between TSP4 and GFAP immunoreactivities in the ipsilateral and contralateral spinal cord $(P<0.001$, ipsilateral $r=0.826$, contralateral $r=0.811$, Fig. 7 ).

\section{DISCUSSION}

TSP4 protein plays an important role in the NP process. It is considered that interfering with TSP4 expression may enable the development of more effective treatment strategies. Our result showed that the combination of cyanocobalamin and morphine is more effective in antinociception and partially decreased the induced TSP4 expression compared to use alone. There is likely a relationship between TSP4 and the effects of the opioid system and vitamin $\mathrm{B}_{12}$, although complex in vivo mechanisms have prevented this relationship from being understood clearly.

In our study, the unilateral CCI of the rat sciatic nerve caused mechanical, thermal hyperalgesia, and tactile allodynia on the 4th postoperative day. Mechanical hyperalgesia and allodynia, but not thermal hyperalgesia, persisted on the 14th postoperative day. There are contradictory reports regarding the persistence of thermal hyperalgesia in the CCI model of rat NP, i.e., some researchers observed thermal hyperalgesia on the 12th-14th postoperative days $[19,20]$. In contrast, others reported that hypersensitivity to thermal stimuli returned to baseline values following sciatic nerve ligation on the 14th postoperative day [21]. Even when the same NP model is used, differences in the type and diameter of the suture material [22], number of ligations, and degree of ligature tightness may lead to variability in behavioral responses [23]. Additionally, roughly $35 \%$ of the variation among individuals in behavioral responses could be attributed to individual differences [24].

In the present study, spinal TSP4 expression increased significantly in saline-injected neuropathic rats on the 4 th postoperative day but not on the 14th postoperative day. Following peripheral nerve injury, increased spinal astrocytic activity and TSP4 expression mediate the development of behavioral hypersensitivity $[25,26]$ and the conversion of acute pain into chronic pain [27]. It has been reported in studies using various NP models such as spinal cord injury [8], spared nerve injury and spinal nerve ligation [28], an infraorbital nerve CCI model [29], and facet joint distraction [30] that TSP4 expression increased in the relevant spinal cord segments during the period when pain thresholds decreased the most, and the increased TSP4 expression returned to normal when the animals recovered from hypersensitivity [7]. Consistent with these studies, our data showed on the 4 th postoperative day that behavioral hypersensitivity is accompanied by increased spinal TSP4 expression. However, although mechanical hypersensitivity persisted on the 14th postoperative day in our study, increased TSP4 expression did not accompany this hypersensitivity. The inconsistency of our results with previous reports suggests that methodological differences in the induction of NP in animals strongly affect the recovery duration from hypersensitivity, obstructing prediction of the synchronicity of behavioral findings with molecular changes.

Our results show that cyanocobalamin, administered for 15 consecutive days to non-operated rats, did not alter the paw nociceptive thresholds, similar to that proposed by Deng et al. [31]. Accordingly, it can be postulated that the intact nervous system is not directly affected by exogenous $\mathrm{B}_{12}$ intake.

In our study, cyanocobalamin failed to improve the pain threshold of rats with NP when administered alone and caused a significant increase in spinal TSP4 expression on the 4th postoperative day. GFAP, an astrocyte marker, and TSP4 immunoreactivities also increased on the 14th postoperative day. Increased astrocyte activation in the spinal cord [32] and upregulated TSP expression at injury sites [33] are associated with the capacity of axons to regenerate. Vitamin $B_{12}$ plays a role in this process by improving nerve conduction, promoting the regeneration of injured nerves, and inhibiting spontaneous ectopic discharges of injured primary sensory neurons [34]. The lack of an antinociceptive effect from cyanocobalamin may be related to the duration of treatment in our study, since vitamin $B_{12}$ can support nerve regeneration due to its long-term healing effect rather than its short-term pain-reducing effect [16]. Increased TSP4 expression along with astrocyte activation suggests that TSP4 may be an important molecule mediating the regenerative effects of vitamin $\mathrm{B}_{12}$.

In this study, low-dose morphine $(2.5 \mathrm{mg} / \mathrm{kg} / \mathrm{day})$ alone did not provide an antinociceptive effect in treating neuropathic rats. High-dose morphine $(5 \mathrm{mg} / \mathrm{kg} /$ day) alone exhibited a partial recovery from tactile allodynia measured by an esthesiometer. However, this effect also decreased over time from the 4 th to the 14 th postoperative day. Inability to obtain the desired antinociceptive effect seems to be compatible with the opioid tolerance which is a reduction of the effectiveness of morphine with the prolongation of the treatment period [35]. Decreased $\mu$-opioid analgesia, increased spinal glial activity, and signs of abnormal pain may occur in both opioid tolerance and NP conditions $[9,36]$. In various studies, it was reported that inhibition of spinal and cortical astrocytic activity in neuropathic rats administered chronic morphine considerably reduced the increased spinal GFAP immunoreactivity [37], exaggerated pain conditions [38], and development of opioid tolerance 
[36]. Administration of both doses of morphine alone to neuropathic rats increased the TSP4 expression on the 4th and 14th postoperative days. Both TSP4 and GFAP immunoreactivities increased in rats treated with high doses of morphine on the 14th postoperative day. In addition to the lack of antinociceptive effect from morphine, the induced spinal astrocytic activity also supports that morphine tolerance occurred in our neuropathic rats. The effects of TSP4 on the development of behavioral hypersensitivity and chronic pain are mediated by the interaction between epidermal growth factor (EGF)-like domains of TSP4 and voltage-gated calcium channel subunit $\alpha_{2} \delta_{1}$ [39]. Opioid receptors exert their effects on various cells through EGF receptor (EGFR) transactivation [40]. Morphine, an opioid receptor agonist, uses the EGF-like domains that came out by hydrolysis of TSP-1 proteins synthesized in astrocytes to activate EGFR [41]. Because all members of the TSP family have EGF-like domains [5], there may be a similar relationship between morphine and TSP4 as well. However, other mechanisms associated with pain can not be ruled out under in vivo conditions. Therefore, it is quite difficult to comment on the direction and course of the relationship between morphine and TSP4, and further studies on this issue are required.

Escalating the dose of opiates to overcome tolerance may lead to serious adverse effects, seriously limiting their therapeutic utilization [35]. The use of opiates in combination with other drugs may allow and maintain analgesic effectiveness without increasing the incidence of adverse effects. Our data showed that the co-administration of cyanocobalamin and morphine produced a significant antihyperalgesic effect, whereas both drugs failed to improve mechanical hyperalgesia assessed by an analgesia meter when administered even at high doses alone. Additionally, this combination reduced the increased TSP4 expression on the 4th and 14th postoperative days and significantly increased TSP4 and GFAP immunoreactivities on the 14th postoperative day. In a limited number of studies performed on mice, it has been reported that cyanocobalamin enhances the antinociceptive effect of morphine, significantly lessens morphine tolerance, delays the onset of morphine dependence, and reduces withdrawal symptoms $[10,31,42]$. Cyanocobalamin increased the antinociceptive effect of morphine in neuropathic rats in the present study. Parallel to this result, the decrease in spinal astrocytic activity also suggests that the tolerance problem may have been overcome.

Immunostaining data showed a linear and strong relationship between ipsilateral and contralateral lumbar dorsal spinal cord GFAP and TSP4 immunoreactivities on the 14th postoperative day. GFAP is a mature astrocyte marker, and increased GFAP expression is accepted as an important indicator of astrocytic reactivation [43]. TSPs are expressed in the reactive astrocytes of the spinal cord following ischemic and mechanical injury [44]. Our results support that TSP4 is synthesized from astrocytes; at the same time, for whatever reason, it seems that spinal astrocyte activation and changes in TSP4 expression are directly proportional.

In our NP groups treated with cyanocobalamin, morphine, and their combination, we detected a significant increase in TSP4 and GFAP immunoreactivities on the contralateral side, although not as much as on the ipsilateral side. In the spinal cord, the expression of gap junction channels is increased under various pathological conditions and may contribute to chronic pain states including NP [45]. Since gap junctions can physiologically connect astrocytes distant from each other, this provides a structural framework for contralateral changes [46].

In conclusion, our data revealed that the combination of cyanocobalamin and morphine reduced both the induced behavioral hypersensitivity and spinal cord TSP4 expression significantly, an effect which was not observed when these drugs were administered alone. The improvement in pain behavior indicates a synergism between these two drugs. Cyanocobalamin enhanced the antinociceptive effect of morphine, most likely by preventing the development of tolerance. Therefore, cyanocobalamin may be a good candidate for combination with morphine in the long-term treatment of NP to achieve a good analgesic effect without the need to increase the morphine dose. However, it should be taken into account that long-term opioid therapy is not recommended for non-cancer chronic pain or pain lasting more than three months. Noble et al. [47] suggested that proper management of a type of strong painkiller (opioids) in well-selected patients with no history of substance addiction or abuse can lead to long-term pain relief for some patients with a minimal (though not zero) risk of developing addiction, abuse, or other serious side effects. However, the evidence supporting these conclusions is weak, and longer-term studies are required to identify the most likely patients to benefit from the treatment [47].

NP can be a complex problem to manage, and sometimes the use of an opioid analgesic can make the difference between bearable and unbearable pain so that patients can get on with their lives. The use of opioids in NP is still valuable because analgesics such as nonsteroidal anti-inflammatory drugs have no proven efficacy against pain of neuropathic origin [48]. Even though opioids cannot be the first line therapy for long-term treatment in NP, interventions that prevent the development of tolerance and dependence on opioids may improve their safety by reducing severe adverse events in well-selected patients. 
It appears that TSP4 protein plays an important role in the NP process. A better understanding of the relationship between the mechanism of action of drugs and TSP4 may enable the development of more effective and/or multimodal treatment strategies. There is a relationship between cyanocobalamin and/or morphine effect and the spinal cord TSP4 expression, and further studies are required to clarify this relationship.

\section{ACKNOWLEDGMENTS}

We would like to thank Gürhan Mutlu from the Department of Pharmacology, School of Medicine, Ege University, for his technical assistance.

\section{CONFLICT OF INTEREST}

No potential conflict of interest relevant to this article was reported.

\section{FUNDING}

This study was supported by the Scientific Research Fund of Ege University (Project No: TGA-2018-20111).

\section{ORCID}

Neslihan Düzenli, https://orcid.org/0000-0001-6764-4423

Sibel Ülker, https://orcid.org/0000-0002-7603-8393

Gülgün Şengül, https://orcid.org/0000-0002-5826-7379

Buse Kayhan, https://orcid.org/0000-0001-7275-5099

Aytül Önal, https://orcid.org/0000-0002-7273-1131

\section{REFERENCES}

1. International Association for the Study of Pain. IASP terminology: neuropathic pain [Internet]. Washington, D.C.: IASP; 2021. Available at: https://www.iasp-pain.org/resources/ terminology/\#neuropathic-pain.

2. Bouhassira D, Attal N. The multiple challenges of neuropathic pain. Neurosci Lett 2019; 702: 6-10.

3. Zilliox LA. Neuropathic pain. Continuum (Minneap Minn) 2017; 23(2, Selected Topics in Outpatient Neurology): 512-32.

4. Meacham K, Shepherd A, Mohapatra DP, Haroutounian S. Neuropathic pain: central vs. peripheral mechanisms. Curr Pain Headache Rep 2017; 21: 28.

5. Adams JC, Lawler J. The thrombospondins. Cold Spring Harb
Perspect Biol 2011; 3: a009712.

6. Bornstein P. Thrombospondins as matricellular modulators of cell function. J Clin Invest 2001; 107: 929-34.

7. Kim DS, Li KW, Boroujerdi A, Yu YP, Zhou CY, Deng P, et al. Thrombospondin- 4 contributes to spinal sensitization and neuropathic pain states. J Neurosci 2012; 32: 8977-87.

8. Zeng J, Kim D, Li KW, Sharp K, Steward O, Zaucke F, et al. Thrombospondin-4 contributes to spinal cord injury-induced changes in nociception. Eur J Pain 2013; 17: 1458-64.

9. Ossipov MH, Lai J, King T, Vanderah TW, Porreca F. Underlying mechanisms of pronociceptive consequences of prolonged morphine exposure. Biopolymers 2005; 80: 319-24.

10. Hosseinzadeh H, Moallem SA, Moshiri M, Sarnavazi MS, Etemad L. Anti-nociceptive and anti-inflammatory effects of cyanocobalamin (vitamin B12) against acute and chronic pain and inflammation in mice. Arzneimittelforschung 2012; 62: 324-9.

11. Xu G, Lv ZW, Feng Y, Tang WZ, Xu GX. A single-center randomized controlled trial of local methylcobalamin injection for subacute herpetic neuralgia. Pain Med 2013; 14: 884-94.

12. Suzuki K, Tanaka H, Ebara M, Uto K, Matsuoka H, Nishimoto $S$, et al. Electrospun nanofiber sheets incorporating methylcobalamin promote nerve regeneration and functional recovery in a rat sciatic nerve crush injury model. Acta Biomater 2017; 53: 250-9.

13. Zhang M, Han W, Zheng J, Meng F, Jiao X, Hu S, et al. Inhibition of hyperpolarization-activated cation current in medium-sized DRG neurons contributed to the antiallodynic effect of methylcobalamin in the rat of a chronic compression of the DRG. Neural Plast 2015; 2015: 197392.

14. Smith HS, Kara SD, Argoff CE. Management of neuropathic pain-current insights and future perspectives. US Neurol 2012; 8: 57-61.

15. Letizia Mauro G, Lauricella L, Vecchio M, Tomasello S, Scaturro D. Efficacy and tolerability of a fixed dose combination of cortex phospholipid liposomes and cyanocobalamin for intramuscular use in peripheral neuropathies. Minerva Med 2019; 110: 455-63.

16. Buesing S, Costa M, Schilling JM, Moeller-Bertram T. Vitamin B12 as a treatment for pain. Pain Physician 2019; 22: E45-52.

17. Bennett GJ, Xie YK. A peripheral mononeuropathy in rat that produces disorders of pain sensation like those seen in man. Pain 1988; 33: 87-107.

18. Lowry OH, Rosebrough NJ, Farr AL, Randall RJ. Protein measurement with the Folin phenol reagent. J Biol Chem 1951; 193: 265-75.

19. Austin PJ, Wu A, Moalem-Taylor G. Chronic constriction of the sciatic nerve and pain hypersensitivity testing in rats. J Vis Exp 2012; (61): 3393.

20. Tsai YC, Sung YH, Chang PJ, Kang FC, Chu KS. Tramadol relieves thermal hyperalgesia in rats with chronic constriction 
injury of the sciatic nerve. Fundam Clin Pharmacol 2000; 14: 335-40.

21. Robinson I, Meert TF. Stability of neuropathic pain symptoms in partial sciatic nerve ligation in rats is affected by suture material. Neurosci Lett 2005; 373: 125-9.

22. Maves TJ, Pechman PS, Gebhart GF, Meller ST. Possible chemical contribution from chromic gut sutures produces disorders of pain sensation like those seen in man. Pain 1993; 54: 57-69.

23. Ro SL, Jacobs JM. The role of the saphenous nerve in experimental sciatic nerve mononeuropathy produced by loose ligatures: a behavioural study. Pain 1993; 52: 359-69.

24. Bell AM, Hankison SJ, Laskowski KL. The repeatability of behaviour: a meta-analysis. Anim Behav 2009; 77: 771-83.

25. Park J, Yu YP, Zhou CY, Li KW, Wang D, Chang E, et al. Central mechanisms mediating thrombospondin-4-induced pain states. J Biol Chem 2016; 291: 13335-48.

26. Obata H, Sakurazawa S, Kimura M, Saito S. Activation of astrocytes in the spinal cord contributes to the development of bilateral allodynia after peripheral nerve injury in rats. Brain Res 2010; 1363: 72-80.

27. Chiang CY, Sessle BJ, Dostrovsky JO. Role of astrocytes in pain. Neurochem Res 2012; 37: 2419-31.

28. Pan B, Yu H, Park J, Yu YP, Luo ZD, Hogan QH. Painful nerve injury upregulates thrombospondin-4 expression in dorsal root ganglia. J Neurosci Res 2015; 93: 443-53.

29. Li KW, Kim DS, Zaucke F, Luo ZD. Trigeminal nerve injuryinduced thrombospondin-4 up-regulation contributes to orofacial neuropathic pain states in a rat model. Eur J Pain 2014; 18: 489-95.

30. Crosby ND, Zaucke F, Kras JV, Dong L, Luo ZD, Winkelstein BA. Thrombospondin-4 and excitatory synaptogenesis promote spinal sensitization after painful mechanical joint injury. Exp Neurol 2015; 264: 111-20.

31. Deng XT, Han Y, Liu WT, Song XJ. B vitamins potentiate acute morphine antinociception and attenuate the development of tolerance to chronic morphine in mice. Pain Med 2017; 18: 1961-74.

32. Qian C, Tan D, Wang X, Li L, Wen J, Pan M, et al. Peripheral nerve injury-induced astrocyte activation in spinal ventral horn contributes to nerve regeneration. Neural Plast 2018; 2018: 8561704.

33. Hoffman JR, O'Shea KS. Thrombospondin expression in nerve regeneration I. Comparison of sciatic nerve crush, transection, and long-term denervation. Brain Res Bull 1999; 48: 413-20.

34. Zhang M, Han W, Hu S, Xu H. Methylcobalamin: a potential vitamin of pain killer. Neural Plast 2013; 2013: 424651.

35. Holtman JR Jr, Crooks PA, Johnson-Hardy J, Wala EP. An- tinociceptive effects and toxicity of morphine-6-O-sulfate sodium salt in rat models of pain. Eur J Pharmacol 2010; 648: 87-94.

36. Raghavendra V, Rutkowski MD, DeLeo JA. The role of spinal neuroimmune activation in morphine tolerance/hyperalgesia in neuropathic and sham-operated rats. J Neurosci 2002; 22: 9980-9.

37. Song P, Zhao ZQ. The involvement of glial cells in the development of morphine tolerance. Neurosci Res 2001; 39: 281-6.

38. Hutchinson MR, Bland ST, Johnson KW, Rice KC, Maier SF, Watkins LR. Opioid-induced glial activation: mechanisms of activation and implications for opioid analgesia, dependence, and reward. ScientificWorldJournal 2007; 7: 98-111.

39. Park JF, Yu YP, Gong N, Trinh VN, Luo ZD. The EGF-LIKE domain of thrombospondin-4 is a key determinant in the development of pain states due to increased excitatory synaptogenesis. J Biol Chem 2018; 293: 16453-63.

40. Phamduong E, Rathore MK, Crews NR, D'Angelo AS, Leinweber AL, Kappera P, et al. Acute and chronic mu opioids differentially regulate thrombospondins 1 and 2 isoforms in astrocytes. ACS Chem Neurosci 2014; 5: 106-14.

41. Ikeda H, Miyatake M, Koshikawa N, Ochiai K, Yamada K, Kiss A, et al. Morphine modulation of thrombospondin levels in astrocytes and its implications for neurite outgrowth and synapse formation. J Biol Chem 2010; 285: 38415-27.

42. Ghazanfari S, Imenshahidi M, Etemad L, Moshiri M, Hosseinzadeh $\mathrm{H}$. Effect of cyanocobalamin (vitamin B12) in the induction and expression of morphine tolerance and dependence in mice. Drug Res (Stuttg) 2014; 64: 113-7.

43. Gomes FC, Paulin D, Moura Neto V. Glial fibrillary acidic protein (GFAP): modulation by growth factors and its implication in astrocyte differentiation. Braz J Med Biol Res 1999; 32: 619-31.

44. Jones EV, Bouvier DS. Astrocyte-secreted matricellular proteins in CNS remodelling during development and disease. Neural Plast 2014; 2014: 321209.

45. Jeon YH, Youn DH. Spinal gap junction channels in neuropathic pain. Korean J Pain 2015; 28: 231-5.

46. Garrison CJ, Dougherty PM, Kajander KC, Carlton SM. Staining of glial fibrillary acidic protein (GFAP) in lumbar spinal cord increases following a sciatic nerve constriction injury. Brain Res 1991; 565: 1-7.

47. Noble M, Treadwell JR, Tregear SJ, Coates VH, Wiffen PJ, Akafomo C, et al. Long-term opioid management for chronic noncancer pain. Cochrane Database Syst Rev 2010; 2010: CD006605.

48. Scholz J, Finnerup NB, Attal N, Aziz Q, Baron R, Bennett MI, et al. The IASP classification of chronic pain for ICD-11: chronic neuropathic pain. Pain 2019; 160: 53-9. 